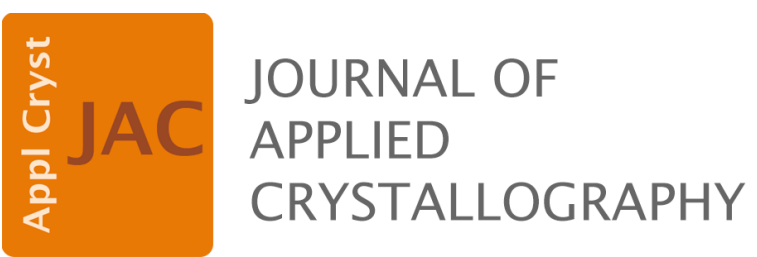

Volume $53(2020)$

Supporting information for article:

A new approach to phason disorder for a decagonal quasicrystal: the moment series expansion of the tiling distribution function for AlCuRh

Ireneusz Bugański, Radoslaw Strzałka and Janusz Wolny 


\section{Appendix $\boldsymbol{A}$}

Here we submit the list of the refined parameters. Table S1 contains parameters for the structure refined without the general Debye-Waller factor. Table S2 contains information about the structure parameters with the general Debye-Waller factor. Coordinates $x$ and $y$ are given in a rhombusspanning base, what means real-structure $\mathbf{r}$ coordinate vector in $2 \mathrm{D}$ plane is obtained as follows: $\mathbf{r}=x \mathbf{d}_{2}+y \mathbf{d}_{3}$, where $\mathbf{d}_{2}$ and $\mathbf{d}_{3}$ are vectors spanning the rhombic tile ( $\mathbf{d}_{2}$ and $\mathbf{d}_{4}$ for thin rhombus). The ' $z$ ' coordinate is given in $\AA$. The letter ' $L$ ' stands for a thick rhomb and the letter ' $S$ ' stands for a thin rhombus. SOF defines site of fraction: 1 means the position is fully occupied, 0 means there is no atom there. The column labeled „Fraction” gives the ratio of atom inside a rhombus depending on it position in a rhombus f. i. atom on the edge is only in half inside a rhombus. Variables $d_{x y}, d_{z z}$ define components of atomic displacement parameters. Table S3 contains refined values of the moments and the shift vector.

Table S1. The list of refined parameters without the general Debye-Waller factor. The model of the expansion of the distribution function into series with moments was used only.

\begin{tabular}{|c|c|c|c|c|c|c|c|c|c|c|c|}
\hline No. & Rhomb & $\mathrm{x}$ & $\mathrm{y}$ & $\mathrm{z}$ & $\mathrm{Al}$ & $\mathrm{Cu}$ & $\mathrm{Rh}$ & SOF & $d_{x y}$ & $d_{z z}$ & Fraction \\
\hline 1 & $\mathrm{~L}$ & 0 & 0 & 1.08176 & 0 & 0 & 1 & 1 & 1.962338 & 3.320625 & 0.2 \\
\hline 2 & $\mathrm{~L}$ & 0.23079 & 0.22949 & 2.33003 & 0 & 0 & 1 & 0.715503 & 1.023047 & 1.834955 & 1 \\
\hline 3 & $\mathrm{~L}$ & 0.31644 & 0.31613 & 1.11511 & 1 & 0 & 0 & 1 & 0.686323 & 0.924195 & 1 \\
\hline 4 & $\mathrm{~L}$ & 0.53334 & 0.53263 & 1.0695 & 0 & 0 & 1 & 1 & 0.593798 & 0.245706 & 1 \\
\hline 5 & $\mathrm{~L}$ & 0.76579 & 0.76364 & 1.24532 & 1 & 0 & 0 & 1 & 6.393461 & 4.896138 & 1 \\
\hline 6 & $\mathrm{~L}$ & 0.85131 & 0.85203 & 1.0695 & 0 & 0 & 1 & 1 & 1.570548 & 0.602379 & 1 \\
\hline 7 & $\mathrm{~L}$ & 0.1506 & -0.00162 & 1.24532 & 1 & 0 & 0 & 1 & 6.89039 & 4.397178 & 1 \\
\hline 8 & $\mathrm{~L}$ & 0.45834 & -0.00008 & 1.0695 & 1 & 0 & 0 & 1 & 0.36323 & 0.173149 & 0.5 \\
\hline 9 & $\mathrm{~L}$ & 0.86032 & 0 & 1.14941 & 0 & 1 & 0 & 1 & 0.950083 & 0.798045 & 0.5 \\
\hline 10 & $\mathrm{~L}$ & 1 & 0 & 2.31168 & 0 & 1 & 0 & 0.727526 & 1.551222 & 0.79255 & 0.3 \\
\hline 11 & $\mathrm{~L}$ & 1 & 0.37897 & 1.08665 & 0 & 0 & 1 & 1 & 0.240539 & 0.201887 & 0.5 \\
\hline 12 & $\mathrm{~L}$ & 1.00001 & 0.85079 & 1.08082 & 1 & 0 & 0 & 1 & 0.130773 & 1.352856 & 0.5 \\
\hline 13 & $\mathrm{~L}$ & 0.27307 & 0.06222 & 1.0695 & 0 & 1 & 0 & 0.5 & 1.975568 & 1.445056 & 1 \\
\hline 14 & $\mathrm{~L}$ & 0.37887 & 0.14687 & 1.0695 & 0 & 0 & 1 & 1 & 0.523905 & 1.076681 & 1 \\
\hline 15 & $\mathrm{~L}$ & 0.52379 & 0.14939 & 1.0695 & 1 & 0 & 0 & 1 & 0.460367 & 0.248695 & 1 \\
\hline 16 & $\mathrm{~L}$ & 0.70879 & 0.08511 & 1.22812 & 1 & 0 & 0 & 1 & 0.154357 & 1.661275 & 1 \\
\hline 17 & $\mathrm{~L}$ & 0.61639 & 0.24171 & 1.0695 & 0 & 0 & 1 & 1 & 0.562472 & 1.076089 & 1 \\
\hline 18 & $\mathrm{~L}$ & 0.77712 & 0.22557 & 1.12214 & 1 & 0 & 0 & 1 & 0.029688 & 0.101955 & 1 \\
\hline 19 & $\mathrm{~L}$ & 0.62133 & 0.38263 & 1.32062 & 1 & 0 & 0 & 1 & 5.468231 & 1.855598 & 1 \\
\hline 20 & $\mathrm{~L}$ & 0.91676 & 0.13472 & 1.0695 & 0 & 1 & 0 & 1 & 0.473769 & 1.421129 & 1 \\
\hline 21 & $\mathrm{~L}$ & 0.9104 & 0.28498 & 1.1051 & 1 & 0 & 0 & 1 & 0.782978 & 2.384478 & 1 \\
\hline 22 & L & 0.85233 & 0.47636 & 1.18412 & 1 & 0 & 0 & 1 & 0.283633 & 1.420402 & 1 \\
\hline 23 & $\mathrm{~L}$ & 0.7556 & 0.62346 & 1.0695 & 0 & 1 & 0 & 1 & 0.25472 & 1.64595 & 1 \\
\hline 24 & $\mathrm{~L}$ & 0.14989 & 0.15 & 2.95038 & 1 & 0 & 0 & 1 & 0.119373 & 2.100859 & 1 \\
\hline 25 & $\mathrm{~L}$ & 0.38165 & 0.38251 & 3.16927 & 0 & 0 & 1 & 1 & 0.262706 & 0.236688 & 1 \\
\hline 26 & $\mathrm{~L}$ & 0.47217 & 0.47442 & 3.04386 & 1 & 0 & 0 & 1 & 0.156699 & 1.806543 & 1 \\
\hline 27 & L & 0.68077 & 0.67825 & 3.15658 & 1 & 0 & 0 & 1 & 1.476138 & 0.955337 & 1 \\
\hline 28 & $\mathrm{~L}$ & 0.91759 & 0.91794 & 3.13195 & 0 & 1 & 0 & 1 & 0.88616 & 1.461657 & 1 \\
\hline 29 & $\mathrm{~L}$ & 1 & 1 & 1.94595 & 0 & 1 & 0 & 0.727526 & 0.251843 & 0.993838 & 0.2 \\
\hline 30 & $\mathrm{~L}$ & 0.23671 & 0 & 3.18986 & 0 & 0 & 1 & 1 & 0.128845 & 0.780386 & 0.5 \\
\hline 31 & $\mathrm{~L}$ & 0.6208 & 0 & 2.59388 & 1 & 0 & 0 & 1 & 0.128715 & 3.244373 & 0.5 \\
\hline
\end{tabular}




\begin{tabular}{|c|c|c|c|c|c|c|c|c|c|c|c|}
\hline ? & $\mathrm{L}$ & 0.76044 & 0 & 3.19262 & 0 & 1 & 0 & 1 & 0.712842 & 1.057451 & 0. \\
\hline & $\mathrm{L}$ & 1 & 0.16316 & 3.13859 & 1 & 0 & 0 & 1 & 0.295849 & 0.598875 & 0.5 \\
\hline & $\mathrm{L}$ & 1 & 0.47243 & 2.97355 & 1 & 0 & 0 & 0.5 & 0.803567 & 4.371369 & \\
\hline & $\mathrm{L}$ & 1 & 0.52702 & 1.27265 & 1 & 0 & 0 & 0.5 & 0.803527 & 4.371386 & \\
\hline & $\mathrm{L}$ & 1 & 0.62212 & 3.15379 & 0 & 0 & & 1 & 0.481152 & 1.356349 & \\
\hline & $\mathrm{L}$ & 0.34182 & 0.08236 & 3.12743 & 1 & 0 & 0 & 1 & 1.166503 & 1.140321 & \\
\hline & $\mathrm{L}$ & 0.4866 & 0.08909 & 3.20850 & 0 & 1 & 0 & 1 & 0.164988 & 0.929828 & \\
\hline & $\mathrm{L}$ & 0.39493 & 0.22859 & 3.16718 & 1 & 0 & 0 & 1 & 0.093377 & 0.57855 & \\
\hline & $\mathrm{L}$ & 0.62975 & 0.13601 & 3.20850 & 1 & 0 & 0 & 1 & 0.117395 & 0.19916 & \\
\hline & $\mathrm{L}$ & 0.52699 & 0.28911 & 2.94759 & 1 & 0 & 0 & 1 & 3.540974 & 3.440886 & \\
\hline & $\mathrm{L}$ & 0.85098 & 0.08966 & 3.18848 & 1 & 0 & 0 & 1 & 3.133096 & 1.52894 & \\
\hline & $\mathrm{L}$ & 0.8469 & 0.2344 & 3.20850 & 0 & 0 & 1 & 1 & 0.309744 & 0.780133 & \\
\hline & $\mathrm{L}$ & 0.6244 & 0.46744 & 3.19304 & 1 & 0 & 0 & 0.401 & 3.197127 & 2.341559 & \\
\hline & $\mathrm{L}$ & 0.77561 & 0.38254 & 2.98843 & 1 & 0 & 0 & 1 & 0.149002 & 5.697984 & \\
\hline & $\mathrm{L}$ & 0.93874 & 0.34443 & 3.19550 & 0 & 1 & 0 & 0.5 & 1.889442 & 0.935681 & \\
\hline & $\mathrm{L}$ & 0.77008 & 0.51763 & 3.18733 & 0 & 0 & 1 & 1 & 0.43795 & 1.152377 & \\
\hline & $\mathrm{L}$ & 0.85393 & 0.61687 & 3.07537 & 1 & 0 & 0 & 1 & 0.099213 & 4.428776 & \\
\hline & $\mathrm{L}$ & 0.90441 & 0.7693 & 3.12954 & 1 & 0 & 0 & 1 & 8477 & 2.445681 & \\
\hline & $\mathrm{L}$ & 0.89546 & 0.67721 & 1.0695 & 1 & 0 & 0 & 1 & 0.047868 & 0.14797 & \\
\hline & $\mathrm{L}$ & 0.05694 & 0.05691 & 3.17273 & 1 & 0 & 0 & 0.4 & 0.743171 & 2.061805 & \\
\hline & $\mathrm{L}$ & 0.4377 & 0.42162 & 1.25634 & 1 & 0 & 0 & 0.5 & 4.074812 & 0.497662 & \\
\hline & $\mathrm{L}$ & 0.67878 & 0.53313 & 1.12725 & 1 & 0 & 0 & 1 & 2.170396 & 1.420691 & \\
\hline & $\mathrm{L}$ & 0.68309 & 0.28712 & 3.20850 & 1 & 0 & 0 & 1 & 0.777904 & 0.548571 & \\
\hline & $\mathrm{L}$ & 0.23406 & 0.23294 & 1.43507 & 0 & 0 & 1 & 0.284497 & 0.232886 & 0.673614 & \\
\hline & $\mathrm{L}$ & 1 & 0 & 1.44081 & 0 & 1 & 0 & 0.21 & 0.704908 & 0.7216 & \\
\hline & $\mathrm{L}$ & 1 & 1 & 2.80664 & 0 & 1 & 0 & 0.21 & 1.143743 & 1.641185 & \\
\hline 1 & $\mathrm{~S}$ & 0 & 0 & 1.08033 & 0 & 0 & 1 & 1 & 1184 & 3445 & \\
\hline 2 & $\mathrm{~S}$ & 0.23934 & 0.23859 & 1.38464 & 1 & 0 & 0 & 1 & 2.074125 & 2.019286 & \\
\hline 3 & $\mathrm{~S}$ & 0.75581 & 0.75753 & 1.10212 & 1 & 0 & 0 & 1 & 2.068138 & 1.404507 & \\
\hline 4 & $\mathrm{~S}$ & 0 & 0.15019 & 1.17113 & 1 & 0 & 0 & 1 & 0.216201 & 0.986457 & \\
\hline 5 & $\mathrm{~S}$ & 0.0015 & 0.46889 & 1.25406 & 1 & 0 & 0 & 1 & 0.792806 & 3.068661 & \\
\hline 6 & $\mathrm{~S}$ & 0 & 0.86019 & 1.11868 & 0 & 1 & 0 & 1 & 0.466248 & 1.361471 & \\
\hline 7 & $\mathrm{~S}$ & -0.00001 & 0.99967 & 2.08582 & 0 & 1 & 0 & 0.727526 & 0.310816 & 2.405977 & \\
\hline Q & $\mathrm{S}$ & 0.38058 & 0.99875 & 1.11084 & 0 & 0 & 1 & 1 & 2.852561 & 0.911862 & \\
\hline 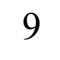 & $\mathrm{S}$ & 0.52823 & 0.99974 & 1.27176 & 1 & 0 & 0 & 0.5 & 6587 & 4.340512 & \\
\hline & $\mathrm{S}$ & 0.83696 & 0.99946 & 1.15351 & 1 & 0 & 0 & 1 & 0.378469 & 0.693835 & \\
\hline 11 & $\mathrm{~S}$ & 0.16872 & 0.39912 & 1.0695 & 0 & 1 & 0 & 1 & 1.610797 & 0.565633 & \\
\hline 11 & $\mathrm{~S}$ & 0.24514 & 0.61355 & 1.126 & 0 & 0 & 1 & 1 & 0.145906 & 0.297515 & \\
\hline 13 & $\mathrm{~S}$ & 0.37734 & 0.47284 & 1.0695 & 1 & 0 & 0 & 1 & 0.957998 & 0.268036 & \\
\hline 14 & $\mathrm{~S}$ & 0.13889 & 0.84788 & 1.25729 & 1 & 0 & 0 & 1 & 0.202989 & 0.543117 & \\
\hline 15 & $\mathrm{~S}$ & 0.24005 & 0.76346 & 1.09056 & 1 & 0 & 0 & 1 & 0.340542 & 0.94394 & \\
\hline 16 & $\mathrm{~S}$ & 0.37963 & 0.61853 & 2.32280 & 0 & 0 & 1 & 1 & 0.498515 & 0.736411 & \\
\hline 17 & $\mathrm{~S}$ & 0.51438 & 0.84515 & 1.0695 & 0 & 1 & 0 & 1 & 2.098595 & 0.302425 & \\
\hline 18 & $\mathrm{~S}$ & 0.61823 & 0.77241 & 1.0695 & 0 & 0 & 1 & 1 & 0.63721 & 0.305768 & \\
\hline 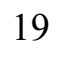 & $\mathrm{S}$ & 0.40002 & 0.40055 & 3.11278 & 0 & 1 & 0 & 1 & 0.874773 & 0.224884 & \\
\hline & $\mathrm{S}$ & 0.99986 & 1.00017 & 1.95488 & 0 & 1 & 0 & 727526 & 1.967803 & 1.410172 & \\
\hline 21 & $\mathrm{~S}$ & 0 & 0.23782 & 3.11695 & 0 & 0 & 1 & 1 & 0.2804 & 0.447393 & \\
\hline & $\mathrm{S}$ & -0.00001 & 0.62222 & 3.02897 & 1 & 0 & 0 & 1 & 0.68737 & 3.389485 & U \\
\hline
\end{tabular}




\begin{tabular}{cccccccccccc}
23 & $\mathrm{~S}$ & 0 & 0.76027 & 3.19218 & 0 & 1 & 0 & 1 & 0.851687 & 1.114681 & 0.5 \\
24 & $\mathrm{~S}$ & 0.14916 & 0.9999 & 3.17807 & 1 & 0 & 0 & 1 & 0.219851 & 1.506929 & 0.5 \\
25 & $\mathrm{~S}$ & 0.46987 & 0.99769 & 2.96778 & 1 & 0 & 0 & 0.5 & 0.222311 & 3.119826 & 1 \\
26 & $\mathrm{~S}$ & 0.6196 & 0.99919 & 3.17206 & 0 & 0 & 1 & 1 & 0.460044 & 0.513857 & 0.5 \\
27 & $\mathrm{~S}$ & 0.15126 & 0.48116 & 3.18107 & 1 & 0 & 0 & 1 & 0.03809 & 0.618578 & 1 \\
28 & $\mathrm{~S}$ & 0.23722 & 0.38274 & 3.10807 & 1 & 0 & 0 & 1 & 1.665278 & 3.479037 & 1 \\
29 & $\mathrm{~S}$ & 0.12486 & 0.59985 & 3.20850 & 0 & 1 & 0 & 1 & 0.100429 & 0.704334 & 1 \\
30 & $\mathrm{~S}$ & 0.24029 & 0.86589 & 3.20850 & 1 & 0 & 0 & 0.5 & 0.418107 & 0.164773 & 1 \\
31 & $\mathrm{~S}$ & 0.3753 & 0.76109 & 3.16621 & 1 & 0 & 0 & 1 & 1.905218 & 0.388858 & 1 \\
32 & $\mathrm{~S}$ & 0.53015 & 0.62845 & 3.07912 & 1 & 0 & 0 & 1 & 0.065106 & 1.241759 & 1 \\
33 & $\mathrm{~S}$ & 0.62659 & 0.85681 & 3.04614 & 1 & 0 & 0 & 1 & 4.40071 & 1.471342 & 1 \\
34 & $\mathrm{~S}$ & 0.77576 & 0.86038 & 3.19667 & 0 & 1 & 0 & 1 & 0.538974 & 1.651472 & 1 \\
35 & $\mathrm{~S}$ & 0.09738 & 0.14509 & 3.17968 & 1 & 0 & 0 & 0.4 & 1.954451 & 1.379616 & 1 \\
36 & $\mathrm{~S}$ & -0.00001 & 1.00007 & 1.48033 & 0 & 1 & 0 & 0.21 & 1.569291 & 1.911193 & 0.1 \\
37 & $\mathrm{~S}$ & 1 & 0.99971 & 2.77161 & 0 & 1 & 0 & 0.21 & 1.188144 & 1.752723 & 0.4 \\
38 & $\mathrm{~S}$ & 0.39393 & 0.64175 & 2.17896 & 0 & 1 & 0 & 0.037493 & 2.519809 & 1.837286 & 1 \\
\hline
\end{tabular}

Table S2. The list of refined parameters, where the general Debye-Waller factor was used together with the model of the expansion of the distribution function into series with moments.

\begin{tabular}{cccccccccccc}
\hline No. & Rhomb & $\mathrm{x}$ & $\mathrm{y}$ & $\mathrm{z}$ & $\mathrm{Al}$ & $\mathrm{Cu}$ & $\mathrm{Rh}$ & $\mathrm{SOF}$ & $d_{x y}$ & $d_{z}$ & Fraction \\
\hline 1 & $\mathrm{~L}$ & 0.00000 & 0.00000 & 1.08177 & 0 & 0 & 1 & 1 & 1.962555 & 3.320889 & 0.2 \\
2 & $\mathrm{~L}$ & 0.23072 & 0.22962 & 2.33293 & 0 & 0 & 1 & 0.715503 & 1.022432 & 1.834487 & 1 \\
3 & $\mathrm{~L}$ & 0.31695 & 0.31700 & 1.11375 & 1 & 0 & 0 & 1 & 0.685871 & 0.923583 & 1 \\
4 & $\mathrm{~L}$ & 0.53284 & 0.53234 & 1.06950 & 0 & 0 & 1 & 1 & 0.593412 & 0.245402 & 1 \\
5 & $\mathrm{~L}$ & 0.76500 & 0.76434 & 1.24425 & 1 & 0 & 0 & 1 & 6.393952 & 4.896339 & 1 \\
6 & $\mathrm{~L}$ & 0.85185 & 0.85192 & 1.06950 & 0 & 0 & 1 & 1 & 1.570487 & 0.602274 & 1 \\
7 & $\mathrm{~L}$ & 0.15000 & -0.00162 & 1.20070 & 1 & 0 & 0 & 1 & 6.890803 & 4.397266 & 1 \\
8 & $\mathrm{~L}$ & 0.45853 & -0.00008 & 1.06950 & 1 & 0 & 0 & 1 & 0.362759 & 0.172744 & 0.5 \\
9 & $\mathrm{~L}$ & 0.86033 & 0.00000 & 1.15472 & 0 & 1 & 0 & 1 & 0.950021 & 0.797865 & 0.5 \\
10 & $\mathrm{~L}$ & 1.00000 & 0.00000 & 2.31330 & 0 & 1 & 0 & 0.727526 & 1.550725 & 0.792013 & 0.3 \\
11 & $\mathrm{~L}$ & 1.00000 & 0.37757 & 1.08982 & 0 & 0 & 1 & 1 & 0.240449 & 0.201773 & 0.5 \\
12 & $\mathrm{~L}$ & 1.00001 & 0.85062 & 1.08040 & 1 & 0 & 0 & 1 & 0.130573 & 1.352399 & 0.5 \\
13 & $\mathrm{~L}$ & 0.27244 & 0.06221 & 1.06950 & 0 & 1 & 0 & 0.5 & 1.975054 & 1.444674 & 1 \\
14 & $\mathrm{~L}$ & 0.37907 & 0.14696 & 1.06950 & 0 & 0 & 1 & 1 & 0.523759 & 1.076766 & 1 \\
15 & $\mathrm{~L}$ & 0.52344 & 0.14996 & 1.06950 & 1 & 0 & 0 & 1 & 0.459761 & 0.248331 & 1 \\
16 & $\mathrm{~L}$ & 0.71002 & 0.08519 & 1.22725 & 1 & 0 & 0 & 1 & 0.153926 & 1.66094 & 1 \\
17 & $\mathrm{~L}$ & 0.61751 & 0.24151 & 1.06950 & 0 & 0 & 1 & 1 & 0.562051 & 1.076258 & 1 \\
18 & $\mathrm{~L}$ & 0.77728 & 0.22675 & 1.12141 & 1 & 0 & 0 & 1 & 0.029324 & 0.101394 & 1 \\
19 & $\mathrm{~L}$ & 0.62163 & 0.38247 & 1.31687 & 1 & 0 & 0 & 1 & 5.46856 & 1.855246 & 1 \\
20 & $\mathrm{~L}$ & 0.91694 & 0.13456 & 1.06950 & 0 & 1 & 0 & 1 & 0.473582 & 1.420956 & 1 \\
21 & $\mathrm{~L}$ & 0.91055 & 0.28473 & 1.10466 & 1 & 0 & 0 & 1 & 0.782528 & 2.384263 & 1 \\
22 & $\mathrm{~L}$ & 0.85227 & 0.47563 & 1.18122 & 1 & 0 & 0 & 1 & 0.283281 & 1.419878 & 1 \\
23 & $\mathrm{~L}$ & 0.75445 & 0.62350 & 1.06950 & 0 & 1 & 0 & 1 & 0.254415 & 1.64572 & 1 \\
24 & $\mathrm{~L}$ & 0.15021 & 0.14998 & 2.95342 & 1 & 0 & 0 & 1 & 0.119 & 2.100564 & 1 \\
25 & $\mathrm{~L}$ & 0.38135 & 0.38146 & 3.16200 & 0 & 0 & 1 & 1 & 0.26272 & 0.236617 & 1 \\
26 & $\mathrm{~L}$ & 0.47251 & 0.47459 & 3.04559 & 1 & 0 & 0 & 1 & 0.156351 & sty.62 & 1 \\
27 & $\mathrm{~L}$ & 0.68037 & 0.67935 & 3.15704 & 1 & 0 & 0 & 1 & 1.475795 & 0.954846 & 1
\end{tabular}




\begin{tabular}{|c|c|c|c|c|c|c|c|c|c|c|c|}
\hline 28 & $\mathrm{~L}$ & 0.91790 & 0.91823 & 3.13133 & 0 & 1 & 0 & 1 & 0.885958 & 1.461404 & 1 \\
\hline 29 & $\mathrm{~L}$ & 1.00000 & 1.00000 & 1.94560 & 0 & 1 & 0 & 0.727526 & 0.251383 & 0.993351 & 0.2 \\
\hline 30 & $\mathrm{~L}$ & 0.23762 & 0.00000 & 3.19087 & 0 & 0 & 1 & 1 & 0.128639 & 0.779647 & 0.5 \\
\hline ; & $\mathrm{L}$ & 0.62074 & 0.00000 & 2.59534 & 1 & 0 & 0 & 1 & 0.128151 & 3.244416 & 0.5 \\
\hline & $\mathrm{L}$ & 0.76054 & 0.00000 & 3.19213 & 0 & 1 & 0 & 1 & 0.712662 & 1.057467 & 0.5 \\
\hline & $\mathrm{L}$ & 1.00000 & 0.16291 & 3.13973 & 1 & 0 & 0 & 1 & 0.295324 & 0.59833 & 0.5 \\
\hline & $\mathrm{L}$ & 1.00000 & 0.47211 & 2.97508 & 1 & 0 & 0 & 0.5 & 0.802943 & 4.371469 & 1 \\
\hline 3 & $\mathrm{~L}$ & 1.00000 & 0.52438 & 1.27098 & 1 & 0 & 0 & 0.5 & 0.802951 & 4.371475 & 1 \\
\hline 36 & $\mathrm{~L}$ & 1.00000 & 0.61892 & 3.15110 & 0 & 0 & 1 & 1 & 0.480911 & 1.356511 & 0.5 \\
\hline 37 & $\mathrm{~L}$ & 0.34261 & 0.08240 & 3.12811 & 1 & 0 & 0 & 1 & 1.166048 & 1.139845 & 1 \\
\hline 8 & $\mathrm{~L}$ & 0.48685 & 0.08883 & 3.20850 & 0 & 1 & 0 & 1 & 0.164686 & 0.929916 & 1 \\
\hline 39 & $\mathrm{~L}$ & 0.39477 & 0.22879 & 3.16833 & 1 & 0 & 0 & 1 & 0.093106 & 0.577958 & 1 \\
\hline 40 & $\mathrm{~L}$ & 0.63028 & 0.13557 & 3.20850 & 1 & 0 & 0 & 1 & 0.117177 & 0.198719 & 1 \\
\hline 41 & $\mathrm{~L}$ & 0.52683 & 0.28992 & 2.95012 & 1 & 0 & 0 & 1 & 3.540852 & 3.440819 & 1 \\
\hline 42 & $\mathrm{~L}$ & 0.85074 & 0.08953 & 3.18891 & 1 & 0 & 0 & 1 & 3.1329 & 1.528567 & 1 \\
\hline 43 & $\mathrm{~L}$ & 0.84612 & 0.23450 & 3.20850 & 0 & 0 & 1 & 1 & 0.309457 & 0.780321 & 1 \\
\hline 44 & $\mathrm{~L}$ & 0.62414 & 0.46761 & 3.19341 & 1 & 0 & 0 & 0.401 & 3.197151 & lut.14 & 1 \\
\hline 45 & $\mathrm{~L}$ & 0.77491 & 0.38308 & 2.99038 & 1 & 0 & 0 & 1 & 0.148618 & 5.698335 & 1 \\
\hline 46 & $\mathrm{~L}$ & 0.93871 & 0.34433 & 3.19607 & 0 & 1 & 0 & 0.5 & 1.889184 & 0.935101 & 1 \\
\hline 47 & $\mathrm{~L}$ & 0.77042 & 0.51807 & 3.18814 & 0 & 0 & 1 & 1 & 0.437405 & 1.151756 & 1 \\
\hline 48 & $\mathrm{~L}$ & 0.85407 & 0.61713 & 3.07764 & 1 & 0 & 0 & 1 & 0.098842 & 4.428781 & 1 \\
\hline 49 & $\mathrm{~L}$ & 0.90503 & 0.76950 & 3.13010 & 1 & 0 & 0 & 1 & 1.398154 & 2.445489 & 1 \\
\hline 50 & $\mathrm{~L}$ & 0.89601 & 0.67700 & 1.06950 & 1 & 0 & 0 & 1 & 0.047766 & 0.147751 & 1 \\
\hline 51 & $\mathrm{~L}$ & 0.05675 & 0.05674 & 3.17321 & 1 & 0 & 0 & 0.4 & 0.742652 & 2.061534 & 1 \\
\hline 52 & $\mathrm{~L}$ & 0.44065 & 0.41980 & 1.26099 & 1 & 0 & 0 & 0.5 & 4.075067 & 0.497225 & 1 \\
\hline 53 & $\mathrm{~L}$ & 0.67918 & 0.53267 & 1.12703 & 1 & 0 & 0 & 1 & 2.170258 & 1.420313 & 1 \\
\hline 54 & $\mathrm{~L}$ & 0.68287 & 0.28423 & 3.20850 & 1 & 0 & 0 & 1 & 0.777569 & 0.548037 & 1 \\
\hline 55 & $\mathrm{~L}$ & 0.23372 & 0.23289 & 1.43054 & 0 & 0 & 1 & 0.284497 & 0.232499 & 0.673068 & 1 \\
\hline 56 & $\mathrm{~L}$ & 1.00000 & 0.00000 & 1.43880 & 0 & 1 & 0 & 0.21 & 0.704381 & 0.721129 & 0.3 \\
\hline 57 & $\mathrm{~L}$ & 1.00000 & 1.00000 & 2.80738 & 0 & 1 & 0 & 0.21 & 1.143282 & 1.640866 & 0.2 \\
\hline 1 & $\mathrm{~S}$ & 0.00000 & 0.00000 & 1.08031 & 0 & 0 & 1 & 1 & 2.301513 & 3.703781 & 0.4 \\
\hline 2 & $\mathrm{~S}$ & 0.23925 & 0.23855 & 1.38403 & 1 & 0 & 0 & 1 & 2.073891 & 2.019027 & 1 \\
\hline 3 & $\mathrm{~S}$ & 0.75620 & 0.75740 & 1.10181 & 1 & 0 & 0 & 1 & 2.067833 & 1.404118 & 1 \\
\hline 4 & $\mathrm{~S}$ & 0.00000 & 0.14998 & 1.17081 & 1 & 0 & 0 & 1 & 0.215857 & 0.985983 & 0.5 \\
\hline 5 & $\mathrm{~S}$ & 0.00150 & 0.46866 & 1.25434 & 1 & 0 & 0 & 1 & 0.792284 & 3.068599 & 1 \\
\hline 6 & $\mathrm{~S}$ & 0.00000 & 0.85965 & 1.12014 & 0 & 1 & 0 & 1 & 0.465923 & 1.361343 & 0.5 \\
\hline 7 & $\mathrm{~S}$ & -0.00001 & 1.00003 & 2.08624 & 0 & 1 & 0 & 0.727526 & 0.310357 & 2.405791 & 0.1 \\
\hline 8 & $\mathrm{~S}$ & 0.38095 & 0.99874 & 1.11187 & 0 & 0 & 1 & 1 & 2.85269 & 0.911561 & 0.5 \\
\hline 9 & $\mathrm{~S}$ & 0.52788 & 0.99974 & 1.27072 & 1 & 0 & 0 & 0.5 & 1.52613 & 4.340621 & 1 \\
\hline 10 & $\mathrm{~S}$ & 0.83714 & 0.99945 & 1.15250 & 1 & 0 & 0 & 1 & 0.377943 & 0.693301 & 0.5 \\
\hline 11 & $\mathrm{~S}$ & 0.16938 & 0.39959 & 1.06950 & 0 & 1 & 0 & 1 & 1.61076 & 0.565344 & 1 \\
\hline 12 & $\mathrm{~S}$ & 0.24639 & 0.61400 & 1.12865 & 0 & 0 & 1 & 1 & 0.145697 & 0.297179 & 1 \\
\hline 13 & $\mathrm{~S}$ & 0.37525 & 0.47270 & 1.06950 & 1 & 0 & 0 & 1 & 0.957674 & 0.267666 & 1 \\
\hline 14 & $\mathrm{~S}$ & 0.13846 & 0.84780 & 1.25638 & 1 & 0 & 0 & 1 & 0.202592 & 0.542638 & 1 \\
\hline 15 & $\mathrm{~S}$ & 0.24045 & 0.76370 & 1.08979 & 1 & 0 & 0 & 1 & 0.340047 & 0.943332 & 1 \\
\hline 16 & $\mathrm{~S}$ & 0.37993 & 0.61825 & 2.32755 & 0 & 0 & 1 & 1 & 0.497784 & 0.735565 & 1 \\
\hline 17 & $\mathrm{~S}$ & 0.51593 & 0.84622 & 1.06950 & 0 & 1 & 0 & 1 & 2.097927 & 0.302069 & 1 \\
\hline 18 & $\mathrm{~S}$ & 0.61695 & 0.77207 & 1.06950 & 0 & 0 & 1 & 1 & 0.637137 & 0.305625 & 1 \\
\hline
\end{tabular}




\begin{tabular}{cccccccccccc}
19 & $\mathrm{~S}$ & 0.39997 & 0.40034 & 3.11362 & 0 & 1 & 0 & 1 & 0.874313 & 0.224432 & 1 \\
20 & $\mathrm{~S}$ & 1.00004 & 1.00010 & 1.95436 & 0 & 1 & 0 & 0.727526 & 1.967485 & 1.409761 & 0.4 \\
21 & $\mathrm{~S}$ & 0.00000 & 0.23778 & 3.11991 & 0 & 0 & 1 & 1 & 0.280021 & 0.4468 & 0.5 \\
22 & $\mathrm{~S}$ & -0.00001 & 0.62207 & 3.03007 & 1 & 0 & 0 & 1 & 0.686859 & 3.389429 & 0.5 \\
23 & $\mathrm{~S}$ & 0.00000 & 0.76025 & 3.19206 & 0 & 1 & 0 & 1 & 0.85137 & 1.114516 & 0.5 \\
24 & $\mathrm{~S}$ & 0.14909 & 0.99991 & 3.17856 & 1 & 0 & 0 & 1 & 0.219308 & 1.506535 & 0.5 \\
25 & $\mathrm{~S}$ & 0.46947 & 0.99767 & 2.96861 & 1 & 0 & 0 & 0.5 & 0.221843 & 3.119749 & 1 \\
26 & $\mathrm{~S}$ & 0.62234 & 0.99920 & 3.16936 & 0 & 0 & 1 & 1 & 0.459932 & 0.513729 & 0.5 \\
27 & $\mathrm{~S}$ & 0.15135 & 0.48088 & 3.18135 & 1 & 0 & 0 & 1 & 0.037684 & 0.618106 & 1 \\
28 & $\mathrm{~S}$ & 0.23678 & 0.38273 & 3.10932 & 1 & 0 & 0 & 1 & 1.664907 & 3.478933 & 1 \\
29 & $\mathrm{~S}$ & 0.12441 & 0.60006 & 3.20850 & 0 & 1 & 0 & 1 & 0.100131 & 0.704089 & 1 \\
30 & $\mathrm{~S}$ & 0.24259 & 0.86545 & 3.20850 & 1 & 0 & 0 & 0.5 & 0.41761 & 0.164239 & 1 \\
31 & $\mathrm{~S}$ & 0.37331 & 0.76107 & 3.16720 & 1 & 0 & 0 & 1 & 1.905001 & 0.388341 & 1 \\
32 & $\mathrm{~S}$ & 0.52892 & 0.62756 & 3.07932 & 1 & 0 & 0 & 1 & 0.064662 & 1.241349 & 1 \\
33 & $\mathrm{~S}$ & 0.62712 & 0.85710 & 3.04728 & 1 & 0 & 0 & 1 & 4.400885 & 1.470944 & 1 \\
34 & $\mathrm{~S}$ & 0.77589 & 0.86085 & 3.19657 & 0 & 1 & 0 & 1 & 0.538721 & 1.65162 & 1 \\
35 & $\mathrm{~S}$ & 0.09723 & 0.14471 & 3.18011 & 1 & 0 & 0 & 0.4 & 1.954109 & 1.37921 & 1 \\
36 & $\mathrm{~S}$ & -0.00001 & 1.00002 & 1.47986 & 0 & 1 & 0 & 0.21 & 1.56893 & 1.910925 & 0.1 \\
37 & $\mathrm{~S}$ & 1.00006 & 1.00000 & 2.77228 & 0 & 1 & 0 & 0.21 & 1.187694 & 1.752425 & 0.4 \\
38 & $\mathrm{~S}$ & 0.39384 & 0.64167 & 2.17918 & 0 & 1 & 0 & 0.037493 & 2.519645 & 1.836991 & 1 \\
\hline
\end{tabular}

Table S3. The refined values of the moments and shift vector. The first type refers to the rhombus from the first pentagon from the atomic surface and second type from the second pentagon.

\begin{tabular}{ccccccc}
\hline Case & Rhombus & $r_{x}[\AA]$ & $r_{y}[\AA]$ & $\left\langle x_{\perp}^{2}\right\rangle\left[\AA^{2}\right]$ & $\left\langle y_{\perp}^{2}\right\rangle\left[\AA^{2}\right]$ & $\left\langle x_{\perp} y_{\perp}\right\rangle\left[\AA^{2}\right]$ \\
\hline Without the & Thick 1 & 0.001398 & -0.01405 & 0.010989 & 0.01099 & 0.001745 \\
general & Thick 2 & -0.02888 & -0.04047 & 0.010972 & 0.010984 & -0.00163 \\
$\begin{array}{c}\text { Debye-Waller } \\
\text { factor }\end{array}$ & Thin 1 & 0.010109 & 0.041965 & 0.010984 & 0.010911 & 0.000948 \\
\hline With the & Thin 2 & -0.01741 & 0.130769 & 0.01042 & 0.008896 & 0.001286 \\
general & Thick 1 & -0.03136 & -0.00679 & 0.010963 & 0.006022 & 0.001657 \\
Debye-Waller & -0.04808 & -0.02118 & 0.003645 & 0.008555 & -0.00162 \\
factor & Thin 1 & -0.00872 & 0.03479 & 0.007526 & 0.008818 & 0.000587 \\
\hline
\end{tabular}

\section{Appendix $B$}

Assuming both local phason flips are equally probable (for both classes of rhombuses within the same type) and the probability of flip is constant independently on the orientation or the time we investigated the correlation between the distribution moments for thin and thick rhombus. Correlations can be used to reduce the number of refinable parameters in the model of the quasicrystal based on the $2^{\text {nd }}$ moment approximation. For obtaining the relations the PT set was generated up to 60 unit cells in each direction of the 5D lattice and projected onto the atomic surface. It was subjected to consecutive 
phason flips following the above mentioned suppositions. The uncertainty comes from calculating moments and standard deviation for subsets of phason flipped PT limited to 50, 4440 and 36 unit cells. All the moments are related to the second moment $\left\langle x_{\perp}^{2}\right\rangle$ of the rhombus's distribution. In the end, the relation between the second moment for the thin and thick rhombus was given. The relations are plotted in the Fig. S1, S2 and S3. All the relations are approximated by the linear function. Moments $\left\langle x_{\perp}\right\rangle$ and $\left\langle y_{\perp}\right\rangle$ give the coordinates of the center of distribution. From the perspective of our approach these moments define the displacement vector $\mathbf{r}^{\boldsymbol{o}, \boldsymbol{c}}{ }_{d}$ in formula (6). 


\section{Thick rhombus I}
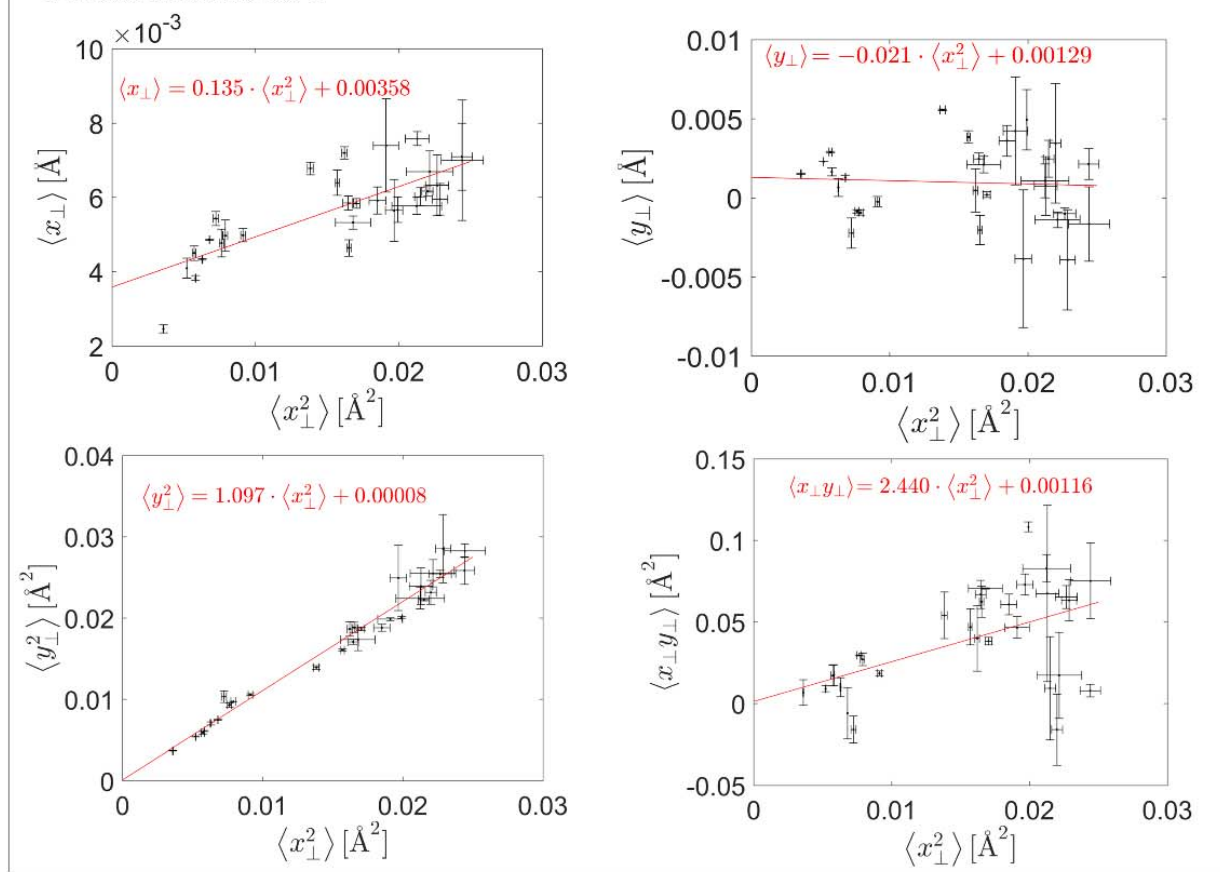

\section{Thin rhombus I}
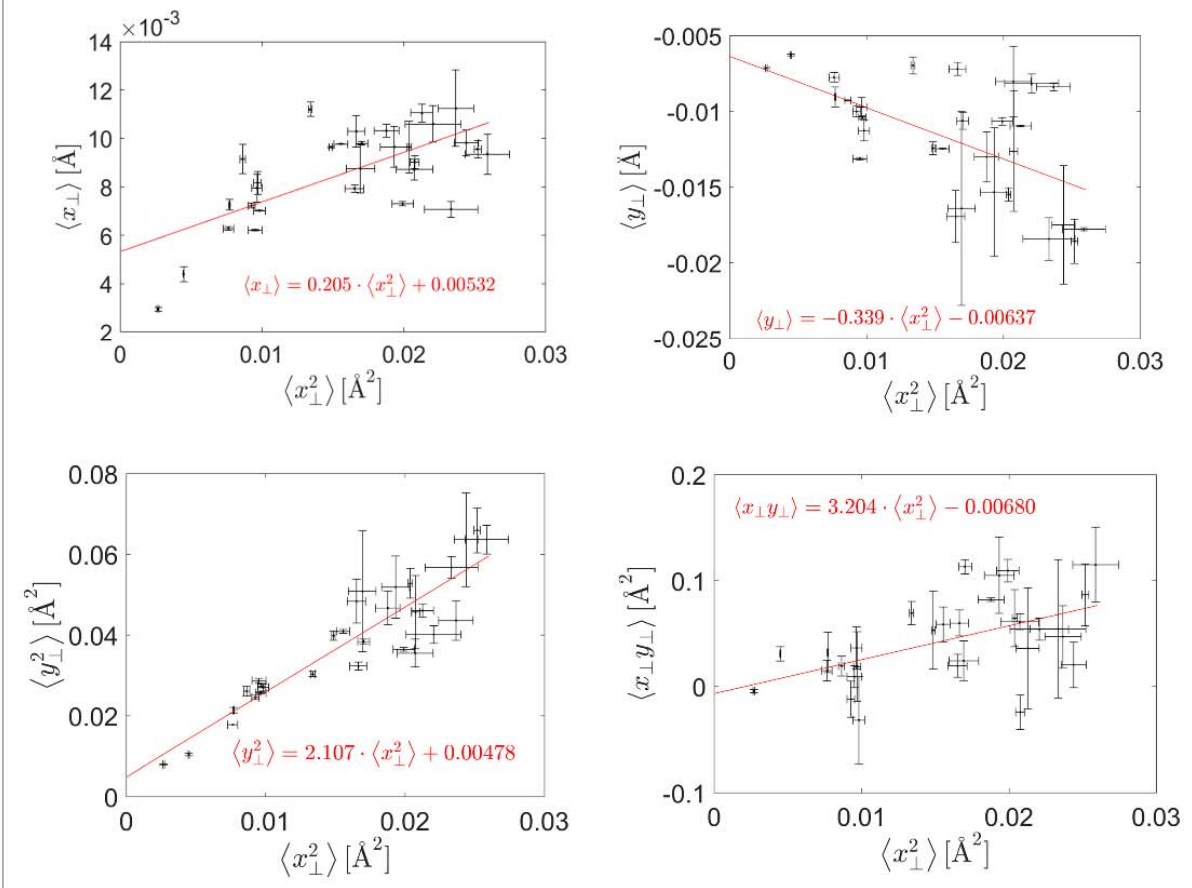

Fig. S1. Relations between moments of the rhombuses distributions. The number "I" refers to rhombuses from the first pentagon (class I - see chapter 1). 

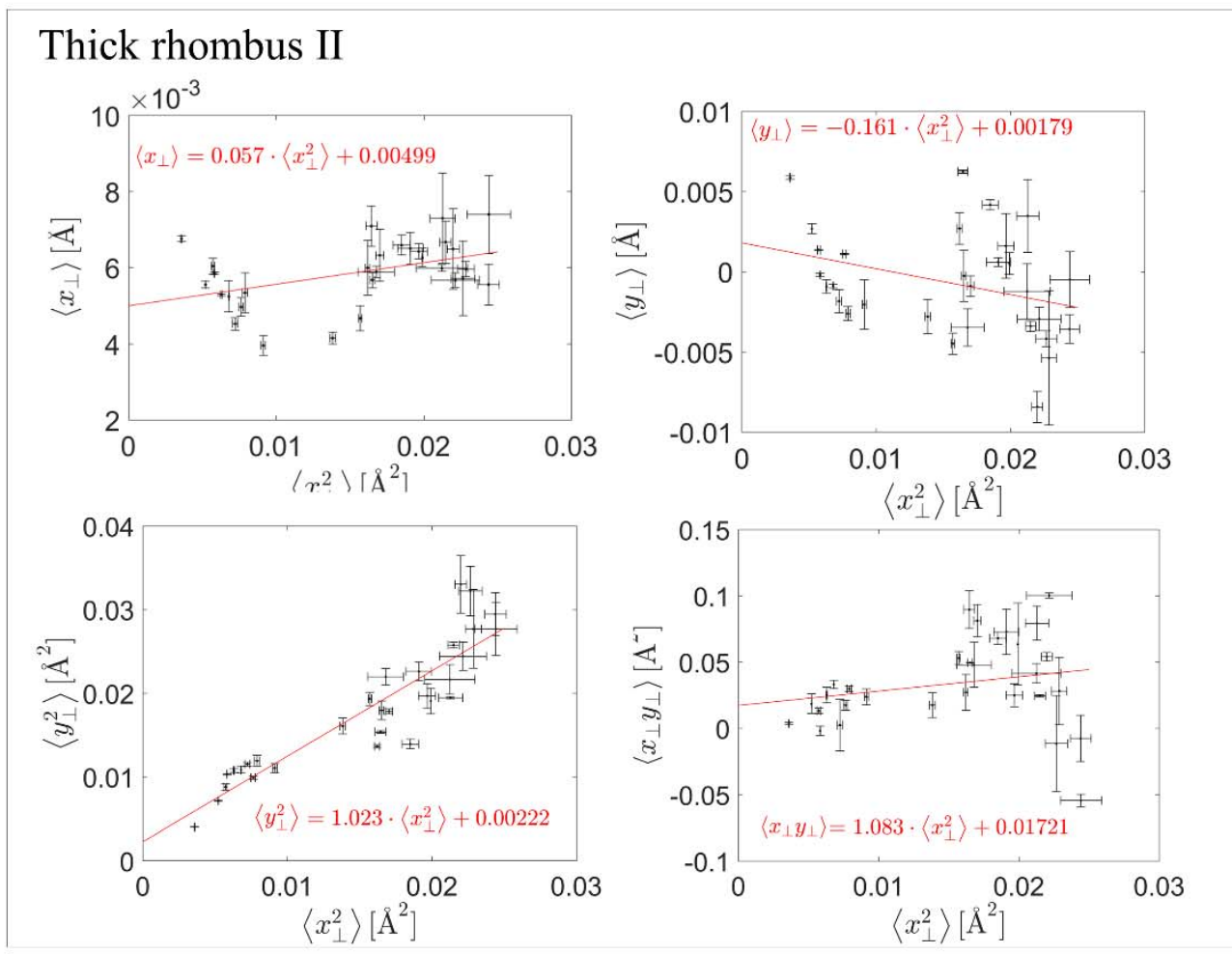

\section{Thin rhombus II}
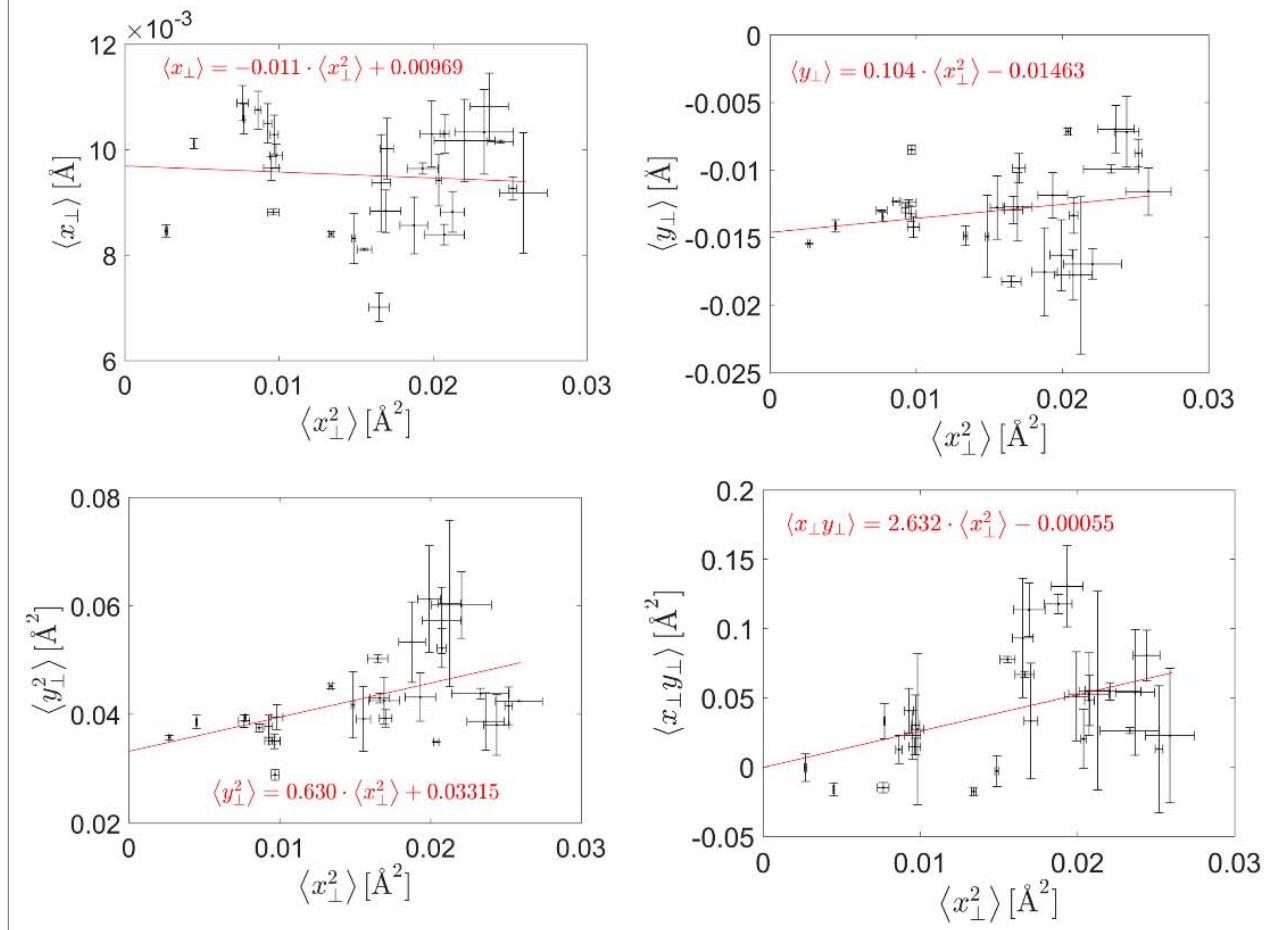

Fig. S2. Relations between moments of the rhombuses distributions. The number "II" refers to rhombuses from the second pentagon (class II - see chapter 1). 


\section{Class I}

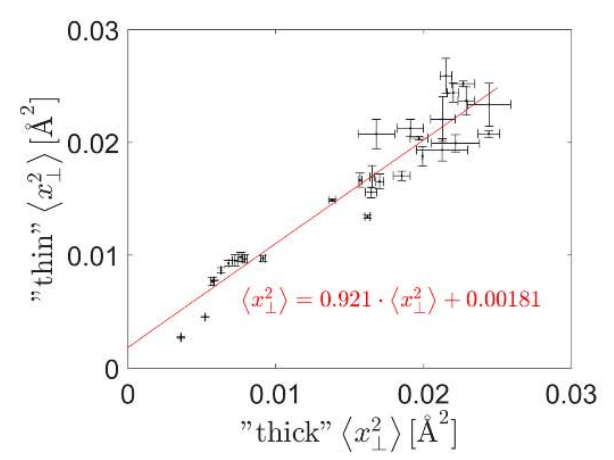

\section{Class II}
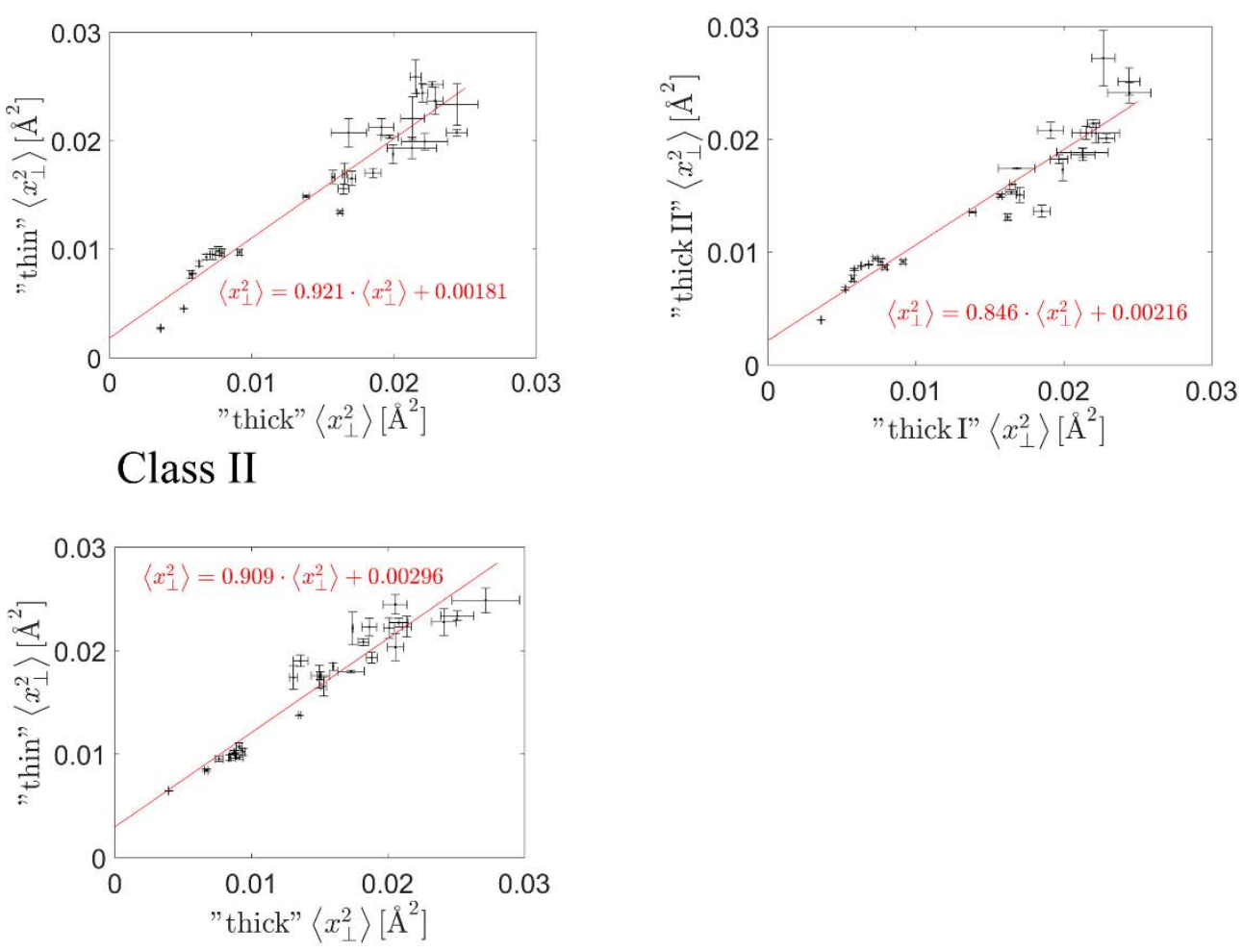

Fig. S3. Relations between the second moments of the distributions. Second moment of the thin rhombus is related to the second moment of the thick rhombus. Afterwards, the second moment of the thick rhombus class II is related to the second moment of the rhombus class I. 